\title{
Chemical reacting transport phenomena and multiscale models for SOFCs
}

\author{
M. Andersson, J. Yuan \& B. Sundén \\ Department of Energy Sciences, Lund University, Sweden
}

\begin{abstract}
Electrochemical reactions at the anode triple phase boundary (TPB) proceed on the basis of the fuel concentration, which depends on transport processes within the porous anode and the heterogeneous reforming chemistry. Microscale modeling is needed to describe these interactions with an acceptable accuracy. The aim of this article is to investigate if it is possible to use a multiscale approach to model solid oxide fuel cells (SOFCs) and combine the accuracy at microscale with for example the calculation speed at macroscale to design SOFCs, based on a clear understanding of transport phenomena and functional requirements. A literature review is made to find out what methods can be used to model SOFCs and also to sort these models after length scale. Couplings between different methods and length scales, i.e., multiscale modeling, are outlined. The SOFC microscale model corresponds in many cases to the atom or molecular level, such as Lattice Bolzmann Method, Density Functional Theory, Molecular Dynamics, Dusty Gas Model, Ficks Model and Stefan-Maxwell Model. SOFC modeling in the mesoscale can be done with Kinetic Monte Carlo. Macroscale models match to the global flow field. Finite Element Method and Finite Volume Method are used to model SOFCs in the macroscale. Multiscale modeling is a promising tool for fuel cell research. COMSOL Multiphysics, based on the Finite Element Method as well as FLUENT, based on the Finite Volume Method, can be used to couple different physical models at different scales. Multiscale modeling increases the understanding for detailed transport phenomena, and can be used to make a correct decision on the specific design and control of operating conditions. It is expected that the development- and production cost will decrease as the understanding of complex phenomena increases.
\end{abstract}

Keywords: SOFC, reactions, transport phenomena, multiscale modelling. 


\section{Introduction}

Fuel cells convert the free energy of a chemical reactant to electrical energy and heat. This is different from a conventional thermal power plant, where the fuel is oxidised in a combustion process combined with a conversion process (thermalmechanical-electrical energy), that takes place after the combustion. Fuel cells can have conversion efficiencies higher than heat engines, since there is no Carnot cycle efficiency limitation [1]. Anode, cathode and electrolyte are the basic building blocks for a fuel cell, their structures decide the efficiency. If pure hydrogen is used no pollution of air and environment occurs, the output from the fuel cell is electricity, heat and water [2].

The reality can often be described at different levels of scale; both length scale and time scale can be varied. This concept can be applied both quantitatively and qualitatively. Modeling a system on a coarser level has two advantages: the modeling is faster and unnecessary details are disregarded [3]. However, at each level of homogenization or scale-up, the risk of losing the key structural information increases [4].

The challenge for the future is to use multiscale modeling to design an optimized nano-structured fuel cell, which is based on functional requirements of a SOFC system [5]. Multiscale modeling is used to understand detailed transport phenomena, to specify the design and control the operating conditions. Combination of micro- and macroscopic analyses provides an engineer a multiscale design tool to understand the detailed transport phenomena and to make a good SOFC design [6]. Fuel cell operation depends on thermal, electrochemical, mechanical and chemical phenomena. A multiscale system-toscience approach is enabled with a multi-physics balance. Cost is reduced, durability is improved and technology development is accelerated [5].

\section{SOFC features}

A fuel cell consists of two electrodes, an anode for fuel and a cathode for oxidant. The electrodes are separated by the electrolyte and connected into an electrically conducting circuit. A gas with fuel or oxidant is transported to the electrode, which

should be permeable via a porous structure. Cells are organised together into stacks [8]. The structure and function (fig. 1) of the different parts of an SOFC are described in this chapter.

An SOFC anode is a mixed ionic-electronic conductor and it consist of either $\mathrm{Ni} / \mathrm{YSZ}$ cermet or $\mathrm{Ni} / \mathrm{Ce}(\mathrm{Sm}, \mathrm{Gd}) \mathrm{O}_{2}$. The electronic conductivity is associated with a conductive percolation path and the ionic conductivity is related to the oxygen vacancies in $\mathrm{YSZ}$ or $\mathrm{Ni} / \mathrm{Ce}(\mathrm{Sm}, \mathrm{Gd}) \mathrm{O}_{2}$. The anode material contains about $30 \%$ wt. of $\mathrm{Ni}$, which works as a catalyst for the oxidation of fuel. An SOFC cathode works in oxygen or air atmosphere. It consist of $\operatorname{Ln}_{1-\mathrm{x}} \mathrm{A}_{\mathrm{x}} \mathrm{MO}_{3}$, where $\mathrm{Ln}=$ lanthanides, $\mathrm{A}=$ alkali-earth metals and $\mathrm{M}=\mathrm{Mn}, \mathrm{Fe}, \mathrm{Co}$ or $\mathrm{Ni}$, such materials are mixed ionic-electronic conductors. The electronic conductivity results from a mixed valency of $\mathrm{M}^{3+} / \mathrm{M}^{4+}$ and the ionic conductivity steams from 
the deviation from stoichiometry in oxygen vacancies. Electrolytes are in contact with the oxidizing atmosphere at the cathode side and with the highly reductive atmosphere at the anode side. Required electronic conductivity is about $10^{-1} \mathrm{~S}$ $\mathrm{cm}^{-1}$ [9]. The nonporous ceramic electrolyte becomes conductive to oxygen ions, $\mathrm{O}^{2-}$, but non-conductive to electrons at the working temperature for SOFCs, 600$1000^{\circ} \mathrm{C}[10]$.

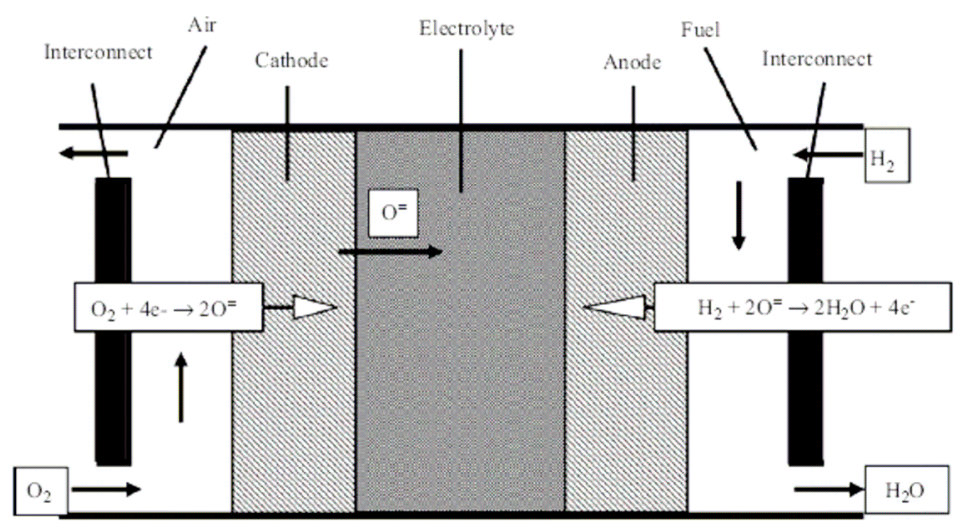

Figure 1: $\quad$ Planar SOFC structure [7].

SOFCs can work with a variety of fuels e.g., hydrogen, carbon monoxide, methane and combinations of these [11]. Oxygen is reduced in the cathode, eqn. (1). The electrons are received from outside the cell and the oxygen ions are delivered to the electrolyte. The electrochemical reactions, eqns. (2)-(3), take place in the anode close to the electrolyte. Methane needs to be reformed, eqn. (4), before the electrochemical reactions [12]. Carbon monoxide can be oxidized in the electrochemical reaction, eqn. (3), but can also react with water eqn. (5). The reactions described here are the overall reactions, more detailed reaction mechanisms can be found in [12-14].

The gas flow in the air and fuel channels can for most cases be considered as one-dimensional and the gas flow is highly viscous and a fully developed velocity profile is established close to the channel entrance. The diffusion in an SOFC is for light gas species around $1 \mathrm{~cm} / \mathrm{s}$, compared to convective velocities of the order of 10 to $100 \mathrm{~cm} / \mathrm{s}$ [11]. Transport in the porous electrodes occurs both in gas- and in solid phase, integrated with the electrochemical reactions. The electrodes are porous and mass transfer is dominated by gas diffusion [15]. The electrolyte has two functions: to transport oxide ions form the electrolyte to the anode and to block electron flow from the anode to the cathode inside the cell [12]. The flow of electronic charge through an outside circuit balances the flow of ionic charge through the electrolyte and electrical power is produced [16]. The interconnect can be assumed to be impermeable for planar SOFC material. Electron transport needs to be considered since current from the SOFC is collected [7].

$$
\mathrm{O}_{2}+4 \mathrm{e}^{-} \Leftrightarrow 2 \mathrm{O}^{2-}
$$




$$
\begin{aligned}
& \mathrm{H}_{2}+\mathrm{O}^{2-} \Leftrightarrow \mathrm{H}_{2} \mathrm{O}+2 \mathrm{e}^{-} \\
& \mathrm{CO}+\mathrm{O}^{2-} \Leftrightarrow \mathrm{CO}_{2}+2 \mathrm{e}^{-} \\
& \mathrm{CH}_{4}+\mathrm{H}_{2} \mathrm{O} \Leftrightarrow 3 \mathrm{H}_{2}+\mathrm{CO} \\
& \mathrm{CO}+\mathrm{H}_{2} \mathrm{O} \Leftrightarrow \mathrm{H}_{2}+\mathrm{CO}_{2}
\end{aligned}
$$

The amount of fuel gases transported to the active surface for electrochemical reactions is governed by different parameters, such as porous microstructure, gas consumption, pressure gradient between the fuel flow duct and the porous anode and inlet conditions [17]. The gas molecules diffuse to the pore walls, where electrocatalytic reaction takes place. The supply of reactant is the rate limiting step, since the gas molecule diffusion coefficient is much smaller than for ions. The charge transfer chemistry at the interface between the electrolyte and the anode proceeds on the basis of the hydrogen concentration. The hydrogen concentration depends on the transport within the porous anode and the heterogeneous reforming chemistry. The concentration of the fuel gases, $\mathrm{CH}_{4}$, $\mathrm{CO}$ and $\mathrm{H}_{2}$, decreases along the length of the fuel channel while the concentration increases for $\mathrm{H}_{2} \mathrm{O}$ and $\mathrm{CO}_{2}$. As a result the current density decreases along the fuel channel [11]. The most active area for reaction inside a SOFC is the TPB. However, not all surface reactions occur there. Depending on the material design, other parts could be as important. The adsorption-desorption reaction may expand onto the electrode surface if the surface diffusion is fast compared to the surface reaction. The oxygen transport path can expand into the electrode/electrolyte boundary if the diffusion along the electrode/electrolyte boundary or the bulk diffusion electrolyte is fast [18].

\section{Models}

Before designing and constructing a model, it is important to specify what one wants to know, how accurate and why. The choice of computational methods must come from a clear understanding of both the information being computed and the chemical system. It is also needed to be aware of what approximations being made and which one being significant [19].

SOFCs can be examined from different points of view; as an electrochemical generator in a viewpoint of electrochemical modeling at continuum level, as a heat and mass exchanger in a viewpoint of fluid dynamics and transport phenomena, as a chemical reactor in viewpoints of chemical reactions depending on fuel composition and heat effects associated with their electrochemical conversion [7].

An SOFC can be described by different length scales: system scale $\left(\sim 10^{2} \mathrm{~m}\right)$, component scale $\left(\sim 10^{1} \mathrm{~m}\right)$, material aspect at the fuel cell/constituent $\left(\sim 10^{-2} \mathrm{~m}\right)$, flow/diffusion morphologies $\left(\sim 10^{-3} \mathrm{~m}\right)$, material structure/interface $\left(\sim 10^{-6} \mathrm{~m}\right)$, and functional material levels $\left(\sim 10^{-9} \mathrm{~m}\right)[5]$.

Not only proper length scales are needed to describe various parts of a SOFC, also different time scales need to be considered. Cell charging and cathode gas thermal diffusion in $10^{-3} \mathrm{~s}$, convective transport in $10^{-1} \mathrm{~s}$, cell heating and anode streamwise thermal diffusion in $10^{3} \mathrm{~s}$ and cathode streamwise thermal diffusion in $10^{4} \mathrm{~s}$ [21]. The relation between time- and length scales can be seen in fig. 2 . 


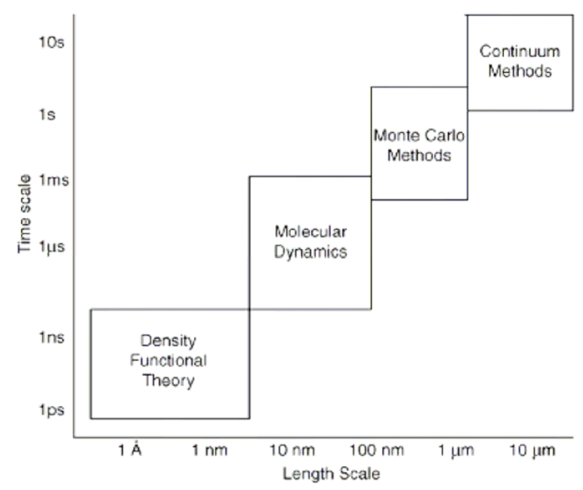

Figure 2: $\quad$ Characteristic time and length scales for various methods [20].

\subsection{Micro-, meso- and macro scale approaches}

Research of natural phenomena is based on different levels of scale; micro-, meso- and macroscales. The microscale model corresponds in many cases to the atom or molecular level when thermo- or fluid dynamics is studied. The microscale does not need to be as small as the size of the molecules. A mesoscale model corresponds to a larger scale than a particle but a smaller one than the facility or the global flow field. Macroscale models match to the global flow field. Microscale modeling is in general more related to theoretical knowledge compared to macroscale modeling that is more related to empirical data. Empirical factors in a macro-model could be based on the result from a micro- or meso model [22].

Instantaneous flow around individual moving particles can be calculated in microscale models. Flows corresponding to calculation cells, larger than particles but smaller than global flow field, are calculated with mesoscale models. Trajectories of individual particles are calculated with particle motion equations for micro scale modeling. The flow field is in mesoscale modeling divided into a number of small cells, but not as small as the particles size [22].

Table 1: $\quad$ Computational methods sorted after scale [20-33].

\begin{tabular}{|l|l|l|}
\hline Microscale & Mesoscale & Macroscale \\
\hline Density Functional Theory (DFT) & Kinetic Monte Carlo & Finite Element Method (FEM) \\
Quantum Chemistry (QC) & (KMC) & Finite Volume Method (FVM) \\
Lattice Bolzmann Method (LBM) & Brownian Dynamics & Finite Differnece Method (FDM) \\
Molecular Dynamics (MD) & Dissipative Particle & Spectral Methods (SM) \\
Mechanistic Models & Dynamics (DPD) & \\
Dusty Gas Model (DGM) & & \\
Ficks Model (FM) & & \\
Stefan-Maxwell Model (SMM) & & \\
\hline
\end{tabular}


Different methods have been developed to describe different scales. Methods that have been used for SOFC modeling are listed in Table 1. The methods are divided into microscale, mesoscale and macroscale. Examples of use for the different methods are described in the following chapters.

\subsubsection{Modeling at the micro scale}

Frayret et al [25] simulated microscopic aspects of oxygen diffusion in the ceriabased material in the ionic conductor with Density Functional Theory (DFT). This methodology is a good tool to study the connection between dopant ionic radius and diffusion at the atomistic scale. DFT is an "ab inito" method where the material properties are described by solutions of the Schrödinger equations. DFT models have a characteristic length scale of $\AA-n m$ and a time scale of ps $-n$ s.

Cheng et al [26] simulated the oxygen ion-hoping phenomenon inside a YSZ electrolyte with Molecular Dynamics (MD). MD can be used to model grain boundary structure, specific heat capacity and molecular structure. Systems up to $10^{5}$ atoms and a time scale on the order of ten ns can be modeled [6].

The Lattice Bolzmann Method (LBM) is used to model mass transport of gases inside the porous anode of an SOFC. The porous structure is based on SEM images, which are converted to digital form. Advantages of the LBM model are that a detailed analysis of mass transfer can be carried out for the actual anode microstructure; this means that tortuosity is not used as a fitting parameter. LBM approach is according to an investigation in [25] accurate enough to model concentration polarization, in 2D, in a SOFC, see Fig. 3(a), where the mole fraction distribution for $\mathrm{H}_{2}$ is shown. By changing the amount of void spaces present in the solid matrix the porosity in the LBM is varied.
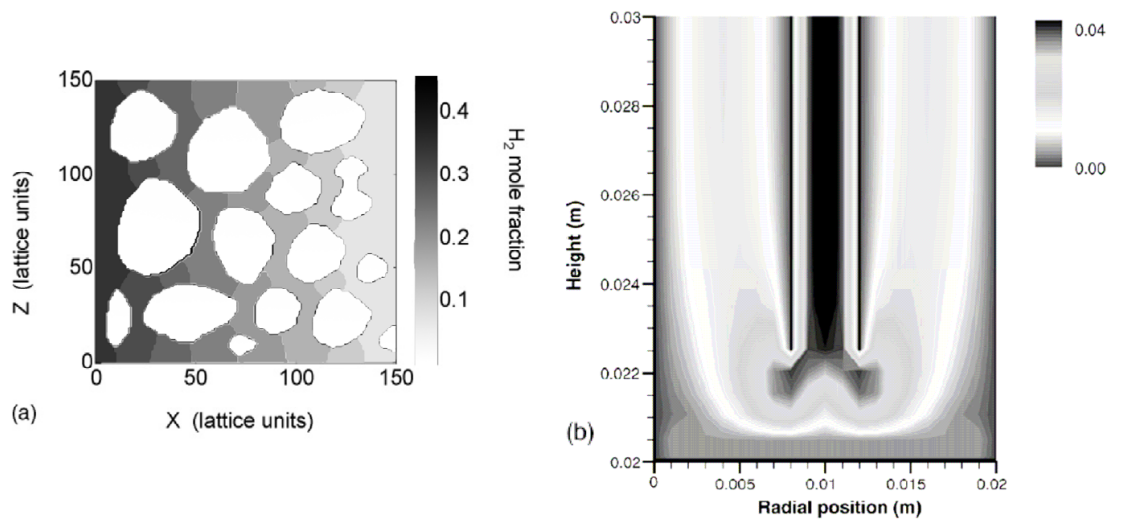

Figure 3: (a) Mole fraction distribution for $\mathrm{H}_{2}$ is calculated with microscale LBM [25]. (b) Dusty gas model is coupled to FVM in FLUENT to calculate the velocity within the anode compartment for a SOFC with tubular design. A modified Butler-Volmer equation and multi-step chemical reactions are parts of the model [14]. 
Dusty Gas model (DGM), Ficks model (FM) and Stefan-Maxwell model (SMM) are developed in [26] in order to predict the concentration over potential inside an SOFC anode. DGM and FM consider molecular diffusion, Knudsen diffusion and the effect of a finite pressure gradient. The flux ratio in DGM depends on the square-root of the gas molecular weight, but it does not for FM. Explicit analytical expressions, which describe fluxes, can be used in FM. The SMM can be seen as a simpler model since it does not consider the Knudsen diffusion. DGM is the most appropriate model for $\mathrm{H}_{2}-\mathrm{H}_{2} \mathrm{O}$ and $\mathrm{CO}-\mathrm{CO}_{2}$ system. However, it is only required to use it when the operating current density is high. $\mathrm{Ni}$ et al $[24,29]$ studied the effect of micro-structural grading on SOFC performance with a DGM, to analyse the coupled phenomena of mass transfer and electrochemical reactions in the SOFC electrodes.

\subsubsection{Modeling at the meso scale}

Modak and Lusk [6] applied Kinetic Monte Carlo (KMC) to simulate the opencircuit voltage and electrical double layer of a doped electrolyte. Discrete time increments of varying size are used to capture diffusion or adsorption in a single step. The physical property data generated by QC and MD can be utilized in the KMC model. Monte Carlo methods have a characteristic length scale of $100 \mathrm{~nm}$ $-\mu \mathrm{m}$ and a time scale of $\mathrm{ms}-\mathrm{s}[23]$.

Huang et al [30] employed COMSOL Multhiphysics (FEM) to model the multhiphysics processes in the SOFC cathode-electrolyte interfaces considering the geometry and detailed distribution of the pores and the ionic conducting phase. The charge transfer rate, electrical conduction and ion conduction are governed on a modeling domain abstracted from actual materials encountered in the application.

\subsubsection{Modeling at the macro scale}

Cheng et al [26] used the Finite Element Methods (FEM) and the commercial software COMSOL Multiphysics to solve the flow equations for macroscopic transport phenomena. Navier-Stokes equations are used to describe the flow conditions in the air and fuel channels and Darcy law describes the flow conditions in the porous layer. FEM has a characteristic time scale of $1 \mathrm{~s}$ and above [23].

A clear relationship between underlying physical conditions and numerical algorithm have made Finite Volume Method (FVM) a popular method for commercial codes such as PHOENICS, FLUENT, CFX and STAR-CD [30]. Pasaogullari and Wang [33] as well as Autossier et al [34] used FLUENT to solve equations for momentum, mass, energy, multicomponent species and electrochemical kinetics for an SOFC. Hussain et al [35] employed the FVM to model the transport of multi-component species inside porous SOFC anodes.

\subsection{Integration issues}

Multiphysics modeling considers interactions, couplings, between two or more physical disciplines. Physical problems can often be described with a set of partial differential equations. The coupled partial differential equations can be 
solved simultaneously in potentially physical domains for each corresponding physical phenomena. Fuel cell operation depends on complex interaction between multi- physics such as multi-phase fluid flow, mass transport, heat transfer and electrochemical reactions [34]. Two basic integration approaches can be found: hierarchical methods, and hybrid and cocurrent method. The hierarchical modeling starts at higher resolution (smaller scale) and properties are extracted and used as input to the next level method. The hierarchical methods are today the most developed methods for multiscale modeling [23]. Three different methods are used for hybrid approaches to describe various regions of the material with the appropriate time and length scale resolutions. The hybrid methods that permit cocurrent simulations are promising for the future development, since only one calculation needs to be performed, however, it requires more computational power compared to hierarchical methods [23].

The particle size in SOFCs is in the sub-micron scale, and the TPBs are in nano-scale. The morphology and properties of these scales are important for the performance of the fuel cell, since they control how much of the Gibbs free energy being available for use. This means that the science at nano-scale is critical to the performance at a system-scale. A robust design and multi-scale analysis consider those nano-details as well as macro system level [5].

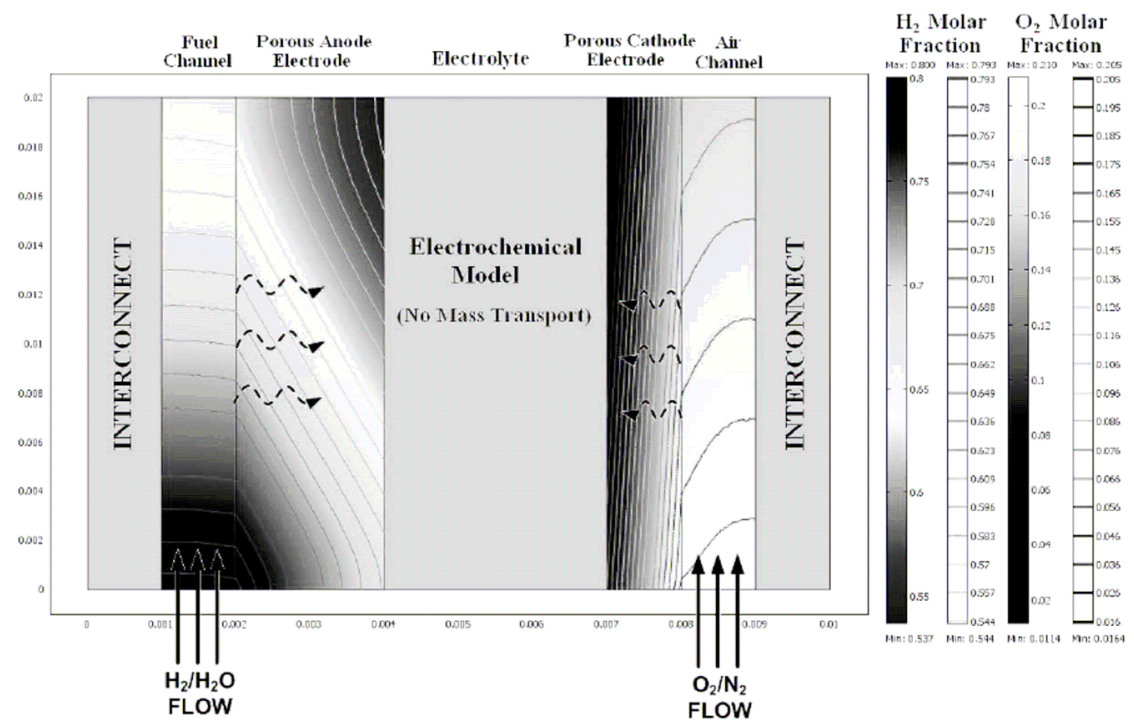

Figure 4: The concentration of $\mathrm{O}_{2}$ and $\mathrm{H}_{2}$ in a SOFC after a multiscale modeling, where Stefan-Maxwell model is used for the flow in air and fuel channels, Dusty-Gas model for transport in porous media, and Butler-Volmer equation for electrochemical phenomena among others [29].

Tseronis et al [31] developed a multidimensional concept, where the microscale DGM is used to describe mass transfer in porous media and FEM 
based COMSOL Multhiphysics is used for the numerical solution of the model equations. Figure 3(b) illustrates the velocity within the anode compartment. DGM is used for porous media transport, Butler-Volmer equation for electrochemicstry, a multi-step model for heterogeneous chemistry and FLUENT is used to couple the different physical descriptions together [14]. Figure 4 illustrates the concentration of $\mathrm{H}_{2}$ and $\mathrm{O}_{2}$ for a multiscale modeling approach in COMSOL Multiphysics, where Stefan-Maxwell model describes the flow in the air and fuel channels, Butler-Volmer equation describes electrochemical phenomena, ionic- and electronic potentials are described by ohmic phenomena and heat transfer is described by radiation, conduction and convection [29].

Cheng et al [26] and Pasaogullari and Wang [33] introduced multi-scaleconcepts; however, they did not mention how the different scales interact with each other. It is frequently stated in literature that data or property constants can be obtained from smaller scale and then used in a model that is made in a larger scale. However, information about the construction of this coupling is rare [14,20-23].

\section{Conclusions}

SOFCs can be described at different length- and time scales. A challenge for the future is to further develop multiscale models for fuel cell designs. Multiscale models can provide a clear understanding of operating conditions, transport and reaction phenomena at the microscale connected to, e.g., conditions in the airand fuel channels at the macroscale.

COMSOL Multiphysics and FLUENT can be used for modeling with multiscale approach. It is possible to couple different physical models, for example models at the microscale, describing transport phenomena inside an anode, to a macroscale model describing the entire fuel cell. It is important to specify what one wants to know and how accurate before designing a model.

Use of multiscale modeling in fuel cell research will lead to an increased power density and also to a lower cost for development and production, i.e., the commercialization of fuel cells will be promoted.

\section{Acknowledgement}

The Swedish Research Council (VR) supports the current research.

\section{References}

[1] Li P.-W., Schaefer L., Chyu M.K., Multiple Transport Processes in Solid Oxide Fuel Cells, Transport Phenomena in Fuel Cells, Sundén B. and Faghri M. (eds.), WIT Press, pp. 1-41, 2005

[2] Yuan J., Faghri M., Sundén B., On Heat and Mass Transfer Phenomena in PEMFC and SOFC and Modelling Approaches, Transport Phenomena in Fuel Cells, Sundén B. and Faghri M. (eds.), WIT Press, pp. 143-174, 2005 
[3] Müller-Plathe F., Coarse-Graining in Polymer Simulation: From the Atomistic to the Mesoscopic Scale and Back, ChemPhysChem, 3, pp. 754769,2002

[4] Gates T., Odegard G., Frankland S., Clancy T., Computational materials: Multi-scale modeling and simulation of nanostructured materials, Comp. Sci. Technol., 65, pp. 2416-2434, 2005

[5] Reifsnider K., Hunag X., Ju G., Solasi R., Multi-scale Modeling Approaches for Functional Nano-composite Materials, J. Mater. Sci., 41, pp. 6751-6759, 2006

[6] Modak A.U., Lusk M.T., Kinetic Monte Carlo Simulation of a Solid-oxide Fuel Cell, Solid State Ionics, 176, pp. 1281-1291, 2005

[7] Kackac S., Pramuanjaroenkij A., Zhou X., A Review of Numerical Modeling of Solid Oxide Fuel Cells, I. J. Hydrogen Energy, 32, pp. 761786, 2007

[8] Beale S.B., Numerical Models for Planar Solid Oxide Fuel Cells, Transport Phenomena in Fuel Cells, Sundén B. and Faghri M. (eds.), WIT Press, pp. 42-82, 2005

[9] Molenda J., Swierczek K., Zajac W., Functional materials for the IT-SOFC, J. Power Sources, 173, pp. 657-670, 2007

[10] Kim S., Lee J., Moon H., Hyun S., Miin J., Kim J., Lee H., Effects of Anode and Electrolyte Microstructures on Performance of Solid Oxide Fuel Cells, J. Power Sources, 169, pp. 265-270, 2007

[12] Zhu H., Kee R., Janardhanan V., Deutschmann O., Goodwin D., Modeling Elementary Heterogeneous Chemistry and Electrochemistry in Solid-Oxide Fuel Cells, J. Electrochem. Soc., 152, pp. A2427-A2440, 2005

[13] Goodenough J.B. Huang Y., Alternative Anode Materials for Solid Oxide Fuel Cells, J. Power Sources, 173, pp. 1-10, 2007

[14] Bessler W.G., Gewies S., Vogler M., A New Framework for Physically Based Modeling of Solid Oxide Fuel Cells, Electrochim. Acta, 53, pp. 1782-1800, 2007

[15] Janardhanan V., Deutschmann O., CFD Analysis of a Solid Oxide Fuel Cell with Internal Reforming, J. Power Sources, 162, pp. 1192-1202, 2006

[16] Nam J. H., Jeon D. H., A Comprehensive Micro-scale Model for Transport and Reaction in Intermediate Temperature Solid Oxide Fuel Cells, Electrochim. Acta, 51, pp. 3446-3460, 2006

[17] Haile S.M. Fuel Cell Materials and Components, Acta Mater., 51, pp. 1981-2000, 2003

[18] Yuan J., Ren F., Sunden B., Analysis of Chemically Reacting Transport Phenomena in an Anode Duct of Intermediate Temperature SOFCs, J. Fuel Cell Sci. Technol., 3, pp. 687-701, 2006

[19] Kawada T., Sase M., Kudo M., Yashiro K., Sato K., Mizusaki J., Sakai N., Horita T., Yamaji K., Yokokawa H., Microscopic Observation of Oxygen Reaction Pathway on High Temperature Electrode Materials, Solid State Ionics, 177, pp. 3081-3086, 2006

[20] Young D., Computational Chemistry A practical guide for Applying Techniques to Real-World Problems, John Wiley \& Sons, 2001 
[22] Karakasidis T., Charitidis C. Multiscale modelling in nanomaterials science, Mater. Sci. Eng., C27, pp. 1082-1089, 2007

[23] Kemm M., Dynamic Solid Oxide Fuel Cell Modelling for Non-steady State Simulation of System Applications, Doctoral thesis, Department of Energy Sciences, Lund University, 2006

[24] Ni, M., Leung M. K. H., Leung D.Y.C., Micro-Scale Modeling of Solid Oxide Fuel Cells with Micro-structurally Grades Electrodes, J. Power Sources, 168, pp. 369-378, 2007

[25] Frayret C., Villesuzanne A., Pouchard M., Matar S., Density Functional Theory Calculations on Microscopic Aspects of Oxygen Diffusion in CeriaBased Materials, I. J. Quantum Chemistry, 101, pp. 826-839, 2005

[26] Cheng C.H., Chang Y.W., Hong C.W. Multiscale Parametric Studies on the Transport Phenomenon of a Solid Oxide Fuel Cell, J. Fuel Cell Sci. Technol., 2, pp. 219-225, 2005

[27] Joshi A.S., Grew K.N., Peracchio A.A., Chiu W.K.S., Lattice Boltzmann Modeling of 2D Gas Transport in a Solid Oxide Fuel Cell Anode, J. Power Sources, 164, pp. 631-638, 2007

[28] Suwanwarangkul R., Croiset E., Fowler M.W., Douglas P.L., Entchev E., Douglas M.A., Performance Comparison of Fick's, Dusty-gas and StefanMaxwell Models to Predict the Concentration Overpotential of a SOFC Anode, J. Power Sources, 122, pp. 9-18, 2003

[29] Ni, M., Leung M. K. H., Leung D.Y.C., Micro-Scale Modeling of a Functionally Graded Ni-YSZ Anode, Chem. Eng. Technol., pp. 287-592, 2007

[30] Huang W., Huang X., Reifsnider K., Meso-Scale Multiphysics Model of SOFC Cathode Processes, COMSOL Users Conference Boston, 2006

[31] Tseronis K., Kookos I., Theodoropoulos K., Modelling and Design of the Solid Oxide Fuel Cell Anode, COMSOL Users Conference Birmingham, 2006

[32] Versteeg H.K., Malalasekera W, An Introduction to Computational Fluid Dynamics, The Finite Volume Method, Pearson, 1995

[33] Pasaogullari U., Wang C.-Y., Computational Fluid Dynamics Modeling of Solid Oxide Fuel Cells, Proceedings of SOFC-VIII, Eds. S.C. Singhal and M. Dokiya, pp. 1403-1412, 2003

[34] Autissier N., Larrain D., Van Herle J., Favrat D., CFD Simulation Tool for Solid Oxide Fuel Cells, J. Power Sources, 131, pp. 131-319, 2004

[35] Hussein M. M., Li X., Dincer I., Mathematical Modeling of Transport Phenomena in Porous SOFC Anodes, Int. J. Thermal Sciences, 46, pp. 4886, 2007

[36] Dennis, B.H., Han Z., Jin W., Wang B.P., Xu L., Aapro, T., Ptchelintsev, A., Reinikainen, T., Multi-Physics Simulation Strategies with Application to Fuel Cell Modeling, 7th. Int. Conf. on Thermal, Mechanical and Multiphysics Simulation and Experiments in Micro-Electronics and MicroSystems, EuroSimE, 2006 